\title{
A multicenter prospective randomized clinical trial assessing implant design modifications after 3 years
}

Drs. S. Raes ${ }^{1}$, C. Stanford ${ }^{2}$, C. Barwacz ${ }^{3}$, D. Cecchinato ${ }^{4}$, J. Brandt', N. Bittner ${ }^{6}$, Prof. dr. H. De Bruyn ${ }^{1}$ ${ }_{1}^{1}$ Ghent University, Faculty of Medicine and Health Sciences, Dental School, Department of Periodontology, De Pintelaan 185, B-9000 Ghent, Belgium ${ }^{2}$ UIC Distinguished Professor and Dean, College of Dentistry, University of Illinois at Chicago, Chicago Illinois, USA.
${ }^{3}$ Assistant Professor, Dows Institute for Dental Research, College of Dentistry, University of lowa, lowa City, lowa, USA

\section{Background}

Implant therapy depends, apart from patientrelated factors, on implant-related factors. Initial implant stability at time of placement is an important clinical factor in implant survival and is considered a prerequisite for early or immediate loading protocols.

\section{Aim}

This RCT evaluates the 3 years outcome of modifications of the OsseoSpeed implant (Dentsply Sirona Implants, Molndal, Sweden) in terms of survival, bone level and peri-implant health. Alterations in the OsseoSpeed EV consisted of changes in dimensions of body shape, drilling procedures, drill design and abutment connection compared to the original OsseoSpeed TX.

\section{Methods and Materials}

120 partially edentulous patients, enrolled in five clinics, were randomized to test (EV) and control (TX). Cemented permanent restorations were placed 9 weeks later on titanium stock abutments. Peri-implant bone level changes, plaque, probing pocket depths and bleeding indices were assessed at restoration placement (baseline), and thereafter at 6, 12 and 36 months. Statistical comparison between EV and TX were done with Mann Whitney U-test with $p<0.05$ as significance level.

\section{Results}

104 subjects (50 EV, 54 TX) were assessed (66 and 75 implants respectively) after 3 years. Three patients discontinued due to failure, 7 dropped out and 6 were not available. The groups were comparable $(p>0.05)$ regarding age, gender, race, smoking habits, tooth loss reason, periodontal history, implant location, length and diameter.

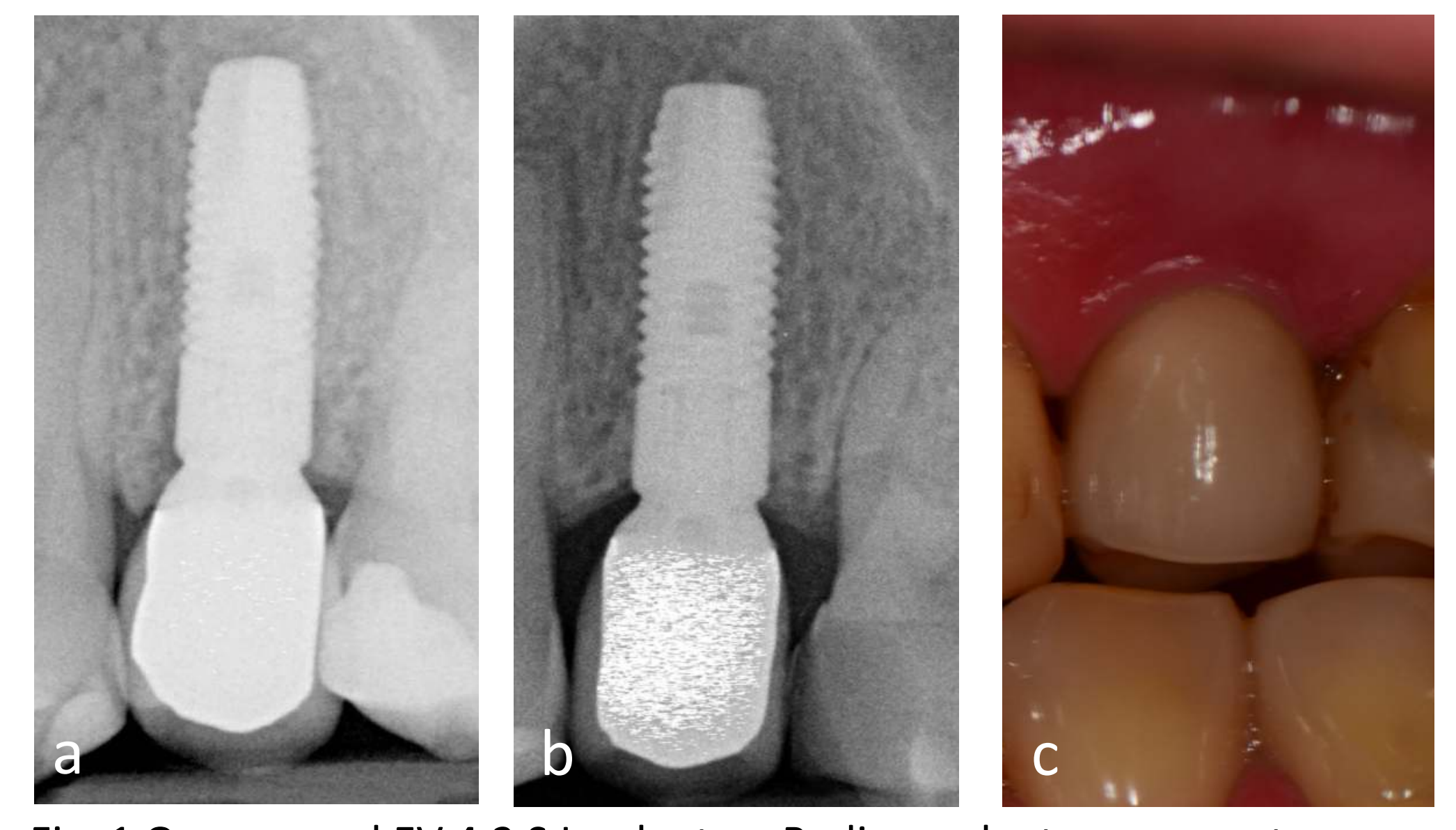

Fig. 1 Osseospeed EV $4.2 \mathrm{~S}$ Implant: a. Radiograph at permanent crown placement; b-c. Radiograph and clinical picture 3 years after crown placement
Mean implant insertion torque was $30 \pm 13 \mathrm{Ncm}$ and $22 \pm 9 \mathrm{Ncm}$ for $\mathrm{EV}$ and $\mathrm{TX}$, indicating improved stability for the modified protocol.

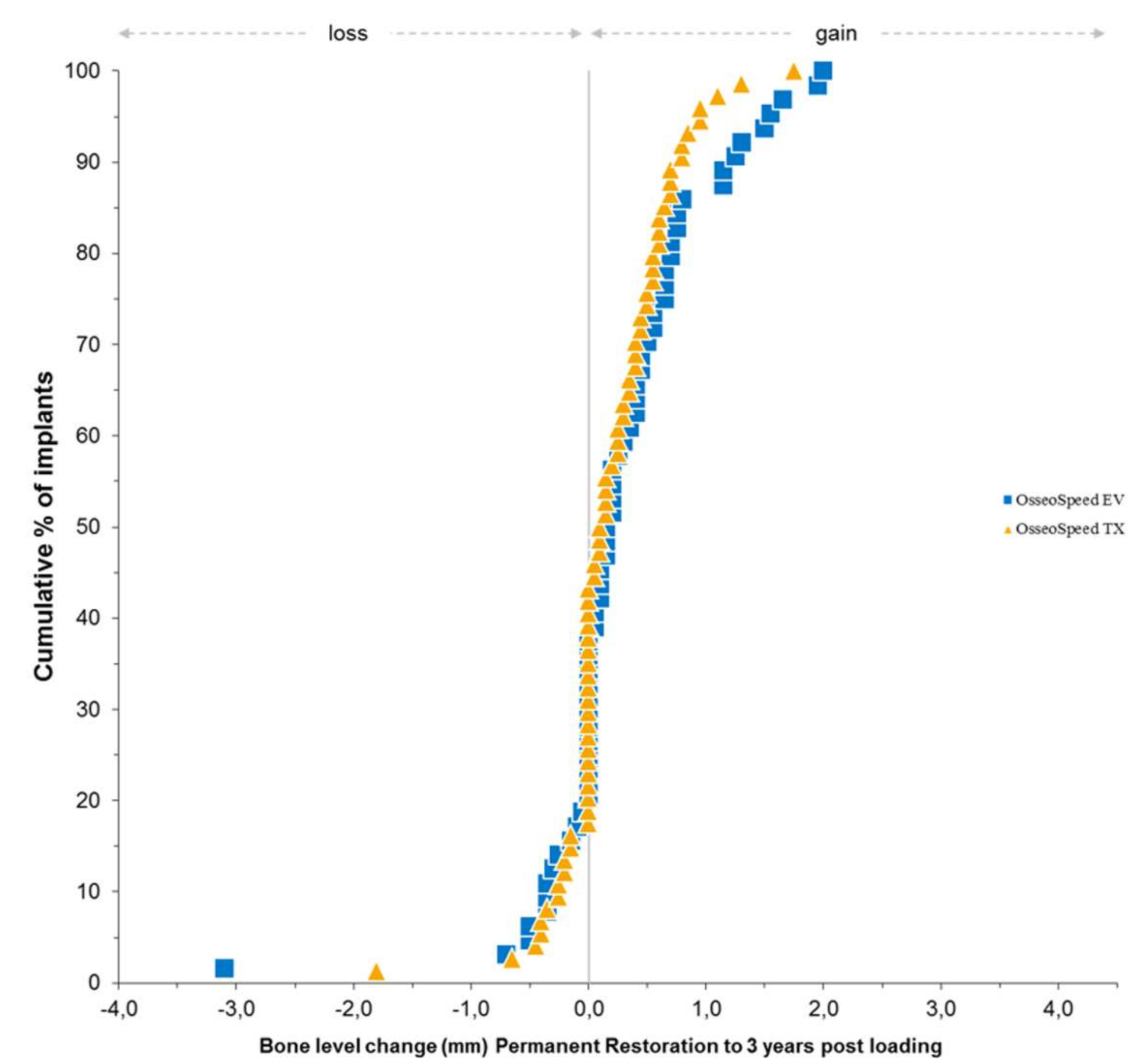

Five of $79 \mathrm{EV}$ implants were lost $(93.6 \%, 4$ prior and 1 post-loading). One of 87 TX implants failed prior to loading (98.9\%). This difference was not statistically significant. Regarding crestal bone change, a mean gain was observed from baseline to 3 years of $0.29 \mathrm{~mm}$ (SD 0.72) and $0.21 \mathrm{~mm}$ (SD 0.48) for EV and TX respectively. Probing pocket depth at 3 years was $2.72 \mathrm{~mm}$ (SD 0.73 ) vs. $2.82 \mathrm{~mm}$ (SD 0.73 ). Plaque was present on $8.3 \%$ and $9.0 \%$ of surfaces, and bleeding on probing remained stable with a mean of $17.4 \%$ and $18.0 \%$. None of the parameters were statistically significantly different between groups $(p>0.05)$.

\section{Conclusions}

Peri-implant health did not differ. A gain in crestal bone levels was observed in both groups but EV implants indicated an improved primary stability.

Although not possible to determine, it cannot be excluded that in three of the four early EV failures, a deviation in the cortical bone preparation may have contributed to the outcome.

Experiences from this study led to modifications of the suggested drilling protocol including widening of the spongious bone preparation to facilitate the implant placement within recommended torque value.

\section{References}

Cecchinato D, Lops D, Salvi GE, Sanz M. A prospective, randomized, controlled study using OsseoSpeed implants placed in maxillary fresh extraction socket: Soft tissues response. Clin

Cecchinato D, Parpaiola A, Lindhe J. A cross-sectional study on the prevalence of marginal bone loss among implant patients. Clin Oral Implants Res 2013;24:87-90.

Noelken R, Neffe BA, Kunkel M, Wagner W. Maintenance of marginal bone support and soft tissue esthetics at immediately provisionalized Osseospeed implants placed into extraction 